\title{
Laser treatment of primary ring-shaped epithelial iris cyst
}

\author{
A. J. BRON,' C. B. WILSON, AND A. R. HILL \\ From the Department of Ophthalmology, University of Oxford, and the !Oxford Eye Hospital, \\ Walton Street, Oxford OX2 6AW
}

SUMmARY This is the first report of a ring-shaped, primary cyst of the iris pigment epithelium. The patient, a 28-year-old woman, presented with angle closure glaucoma. Ocular pressure was controlled medically, and the iris cyst was treated by argon laser photocoagulation. The derivation of the cyst, differential diagnosis, and mechanism of angle closure glaucoma are discussed.

Primary cysts of the iris epithelium are uncommon, and although they may reach a large size are usually located focally. An unusual ring-shaped iris cyst which occupied the whole of the posterior iris surface and presented as angle closure glaucoma is reported here. It was treated successfully by laser photocoagulation.

\section{Case report}

A 28-year-old Caucasian woman presented in the casualty department of the Oxford Eye Hospital on 11 July 1983 with a history of hazy vision in the right eye, associated with rainbows around lights, and pain, occurring over the previous five days. She had vomited on the morning of her first attendance. There was no relevant personal or family history.

Examination in the casualty department showed vision in the right eye to be $6 / 5+3$ and in the left $6 / 4$ with her myopic correction (for refraction). The right eye was injected and showed a diffuse epithelial corneal oedema. A poor view of iris detail was achieved, but the pupil was round, dilated, and poorly mobile to direct and indirect light. Ocular pressures were recorded as right 58 and left $12 \mathrm{mmHg}$. A diagnosis of angle closure glaucoma was made. The patient was admitted and treated with a standard dose of acetazolamide (Diamox) $500 \mathrm{mg}$ intravenously and pilocarpine $4 \%$ drops intensively. The ocular pressure responded rapidly to this regimen, and it was possible to examine the chamber angle, which at this time appeared open though narrow. On the following two days the ocular pressure was 10 $\mathrm{mmHg}$ in the right eye and the patient continued on

Correspondence to Mr A. J. Bron, FRCS, Nuffield Laboratory of Ophthalmology, Walton Strect, Oxford OX2 6AW. treatment with pilocarpine. Because of the unusual presentation the right pupil was dilated on the third day and slit-lamp examination revealed a remarkable $360^{\circ}$ cystic separation of the iris pigment epithelium from the stromal layer in such a way that it resembled a half filled inner tube of a motor tyre showing folds and creases (Figs. 1 and 2). The ocular pressure at this time was noticed to be $14 \mathrm{mmHg}$. Although the lesion appeared cystic, it was not initially possible to confirm its cystic nature by standard transillumination of the iris through the sclera or pupil.

Various investigations were carried out to establish whether the iris lesion was cystic or solid. It was noted that the anterior chamber depth was equal on the two

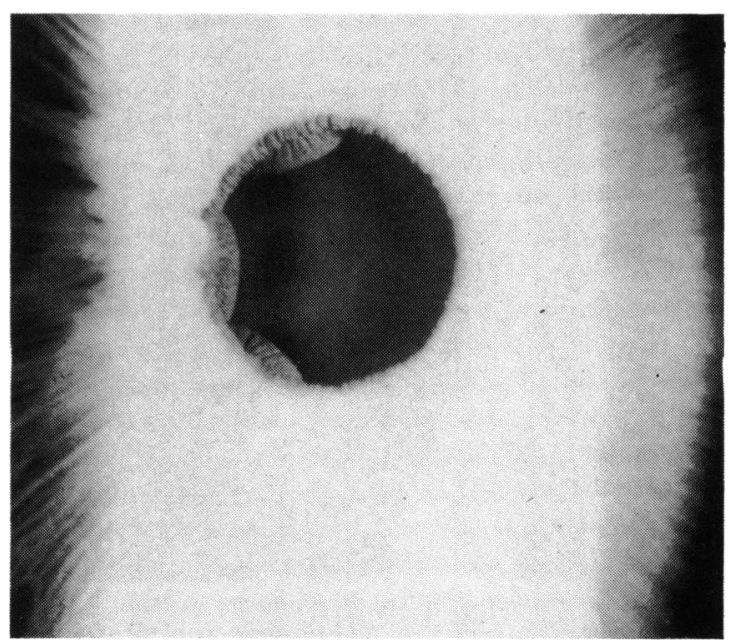

Fig. 1a Low-power view of primary epithelial cyst at the pupil margin. Pupil undilated. 


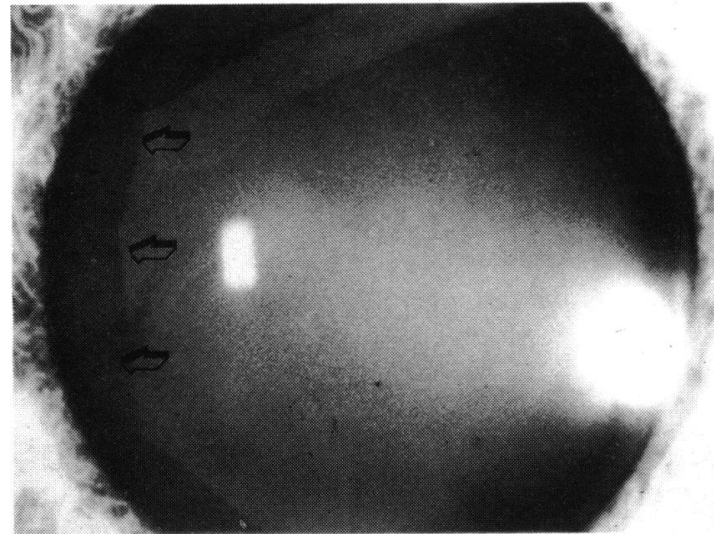

Fig. 1b Appearance of epithelial cyst with pupil dilated. (Inner margin of cyst arrowed).

sides, while the peripheral chamber depth was greatly reduced on the right by the bulging forward of the iris. B-scan of the right eye (Fig. 3) (Sonometrics Ocuscan- 400 with a $100 \mathrm{MH}_{3}$ focused scanning transducer with a $40^{\circ}$ scan sector) showed an expanded lesion with two approximately parallel dense echoes radially within the iris, on both sides of the pupil, bounding an intervening sonolucent zone. A similar appearance was visible on ultrasound examination on all orientations of the scan. The appearances suggested a cystic rather than a solid lesion.

A fluorescein angiogram of the iris showed normal features without a focal or diffuse leakage pattern. Further biomicroscopic examination demonstrated important features. Normally the pigment epithelium of the iris is clearly visible on slit-beam examination

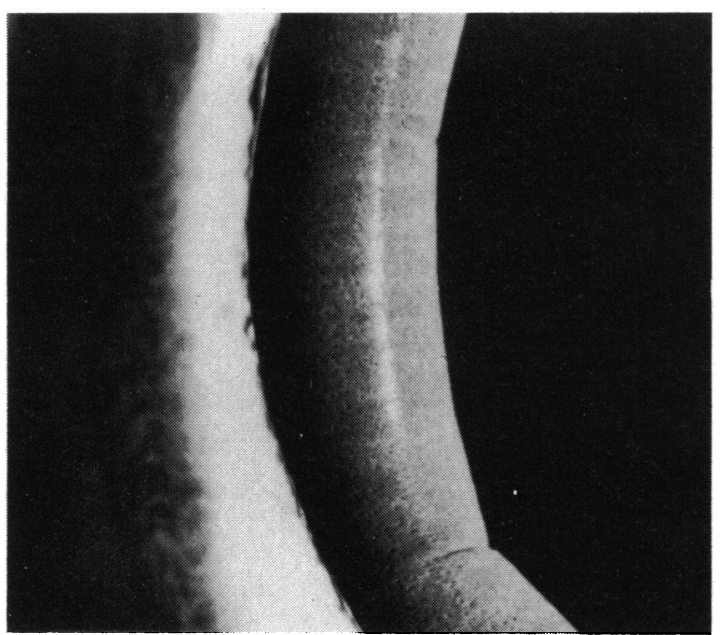

Fig. 2 Macrophotograph of the pigment cyst at the temporal border of the pupil. The posterior surface of the pigment epithelium shows regular depressions. The cyst is distended like a tyre inner tube.

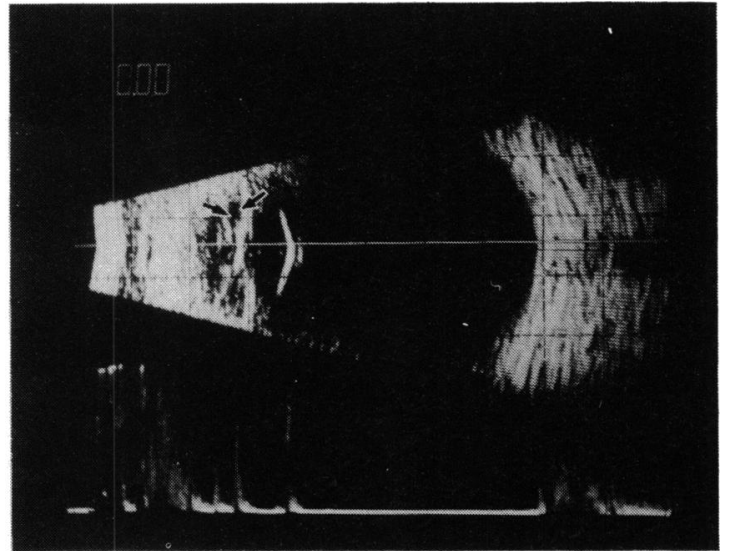

Fig. 3 Combined $A$ and $B$ scan of the right globe. The B . scan shows an expansion of the iris (arrows) with two dense echoes in the sagittal plane encompassing a relatively anechoic region.

of the iris in light coloured irides. The pigment epithelium is seen as a reddish-brown sheet, which may be traced almost from the root of the iris to the sphincter region where the detail becomes obscured by the sphincter (Fig. 4). This normal appearance was seen in the left eye. Examination of the right iris demonstrated that there was no pigment epithelial image visible in the slit beam, but a reddish-brown glow (presumably reflected from the anterior surface of a separated posterior epithelial layer) was visible to the distal side of the slit image on the iris stroma. This was taken to confirm the cystic nature of the lesion, and this feature could be seen nasally, temporally, above and below (Fig. 4). Further confirmation was obtained by shining a bright broad slit of light through the iris stroma and examining the expanded epithelium at the pupil border. In darkness fine diffuse punctate transillumination defects could be seen, again suggesting that the lesion was cystic. This feature could be demonstrated when transilluminating through any quadrant of the iris, and therefore the lesion was thought to be cystic in all parts. Shields ${ }^{12}$ adopts a related technique applying a fibre optic transilluminator over the limbus in the quadrant of the lesion.

As the nature of the lesion was initially uncertain an attempt was made to manage the ocular pressure with medical therapy. While on timolol maleate (Timoptol) $0.25 \%$ drops b.d. with dexamethasone $0 \cdot 1 \%$ drops (G. Maxidex) q.d.s. the ocular pressure rose to $50 \mathrm{mmHg}$, iris bombé was noted to be present, and the angle was closed. The patient was treated with intravenous acetazolamide $500 \mathrm{mg}$ and pilocarpine $4 \%$ drops q.d.s., and on the following day the ocular pressure was $10 \mathrm{mmHg}$ and the angle was open. 

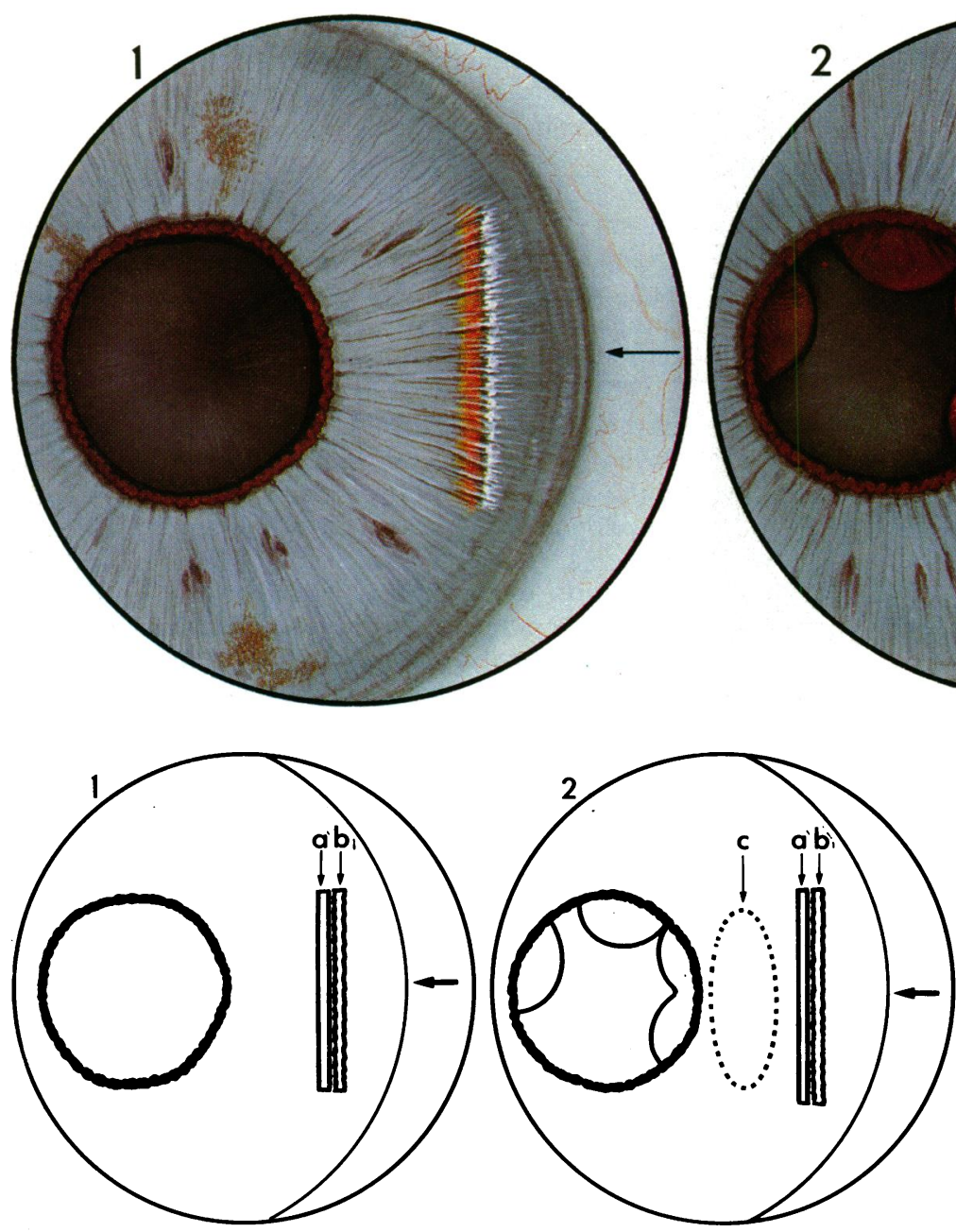

She was discharged on pilocarpine $2 \%$ drops. Two days later she remained asymptomatic, and her regimen was changed to $1 \%$ pilocarpine t.d.s. The patient. returned later the same day with pain in the right eye, reduced vision, and an ocular pressure of $40 \mathrm{mmHg}$. The pupil was mid-dilated, round, and unresponsive to light. Gonioscopy showed iris bombé and closure of three-quarters of the angle. The pressure fell to normal with pilocarpine $4 \%$ drops and the angle fully reopened.

The patient was discharged on a regime of acetazolamide $250 \mathrm{mg}$ t.d.s. and a pilocarpine $2 \%$ drops q.d.s. Since the lesion was thought to be cystic, it was decided that it would be reasonable to attempt to release the fluid contents of the cyst by lasering the iris pigment epithelium visible with the pupil dilated. The patient was given a course of dexamethasone $0 \cdot 1 \%$ drops q.d.s. to the right eye for five days before

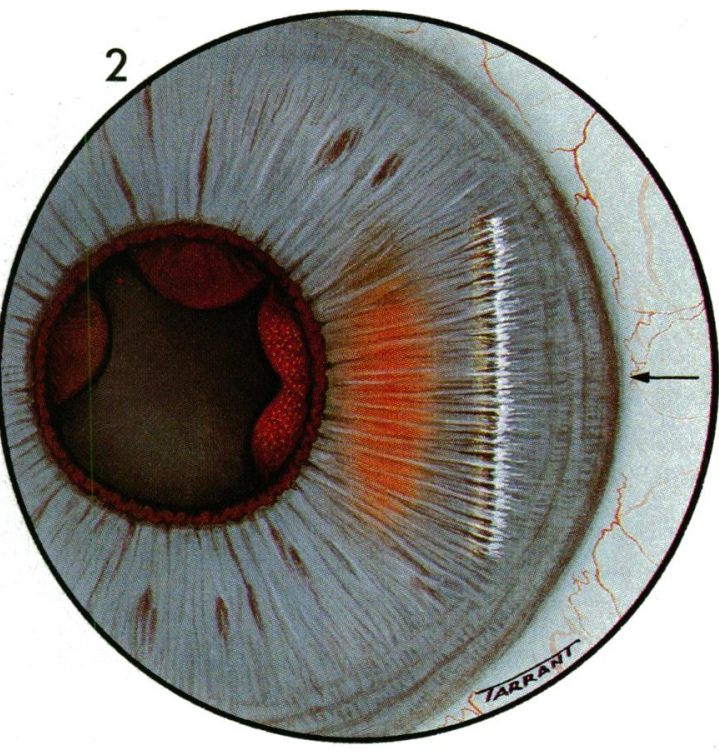

Fig. 4 Diagram to demonstrate the technique of slit examination of the iris epithelium. The examination is carried out in darkness. (1) In the normal, blue iris, the copper coloured pigment epithelium (a) is seen in the slitbeam through the iris stroma. (b). (2) In the presence of an iris cyst a smooth but uncoloured line (a) is seen through the iris stroma (b) at the level of the anterior iris epithelium, but the copper coloured line of the posterior epithelium is lacking. However, a copper coloured glow is visible over the stroma on the pupil side of the slit image representing light reflected from the posterior wall of the cyst (c). In the pupil pinpoints of transilluminated light can be seen scattered over the surface of the cyst.

laser therapy, partly to see the effects of antiinflammatory therapy on cyst size and partly to suppress any postlaser inflammatory response.

On 26 July 1983 argon laser photocoagulation was performed (coherent radiation) under topical anaesthesia and an Abraham lens. A single burn at $10 \mathrm{~mW}$ for $200 \mathrm{~ms}$ and with a $200 \mu \mathrm{m}$ spot was placed in the 9 o'clock meridian close to the pupil margin. It produced a pigment explosion, and with a few more burns it was possible to enter the cyst. At this point it could be seen that the cyst was emptying a clear fluid into the chamber mixed with profuse pigment epi- 


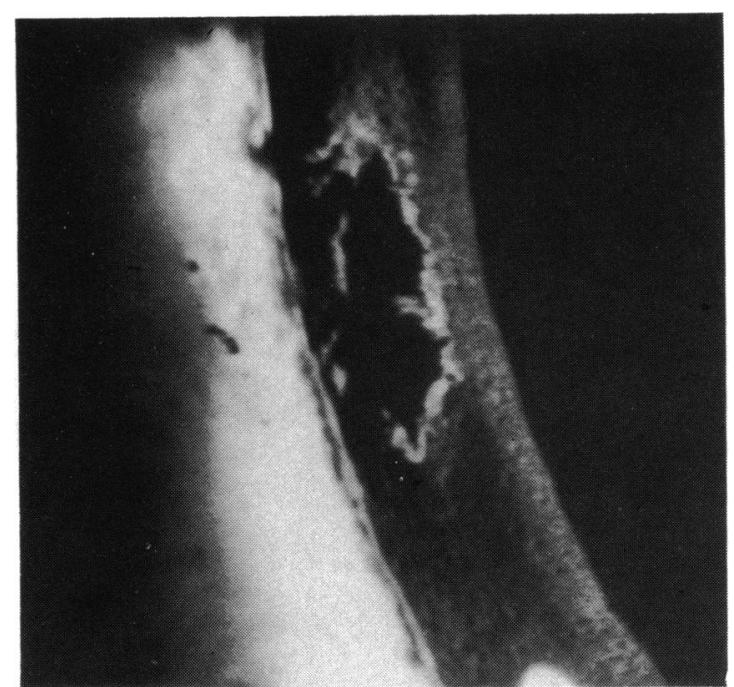

Fig. 5 Macrophotograph of laser hole in the pigment cyst immediately after treatment at the temporal margin of the pupil. Compare with Fig. 2.

thelial debris. There was a gradual flattening of the cyst into a crumpled shell (Figs. 5 and 6). Nineteen burns in all were used to produce an oval pigment epithelial iridotomy and to trim the ragged ends. Postoperatively the ocular pressure rose from 15 to $25 \mathrm{mmHg}$ in a matter of one hour, and this was treated with acetazolamide to bring the pressure down to $12 \mathrm{mmHg}$. The angle was later confirmed to be open, grade 3 all the way round, and identical to that of the left eye. Two months later the pupil size was equal on each side, there was no evidence of a forward convexity of the iris stroma, and the iridotomy site was visible by retroillumination behind the iris sphincter in the 9 o'clock meridian. Pupil dilatation showed that the posterior pigment epithelium was still non-adherent to the anterior epithelium and was present in redundant folds.

\section{Discussion}

The patient described here presented with angle closure glaucoma caused by a primary cyst of the pigment epithelium of the iris. Although this association is well known, Shields ${ }^{1}$ has emphasised that progression to cause serious glaucoma or serious visual complications is uncommon.

The iris pigment epithelium is composed of two layers which are derived from the outer and inner layers of the anterior extremity of the embryonic optic cup. These layers become apposed during development, apex to apex, the anterior epithelium showing relatively sparse pigment granules, while the posterior epithelium is heavily pigmented. The anterior epithelial cells are of interest in that their basal (external) portion gives rise to the ribbon-like smooth muscle processes of the dilator muscle, which project into the overlying iris stroma. The apical portion of the anterior epithelial cells show numerous microvilli, which interdigitate with the similar microvilli of the apical portion of the cells of the posterior pigment epithelium..$^{2}{ }^{3}$ The apical or apposed surfaces of each epithelial layer are connected by desmosomes and tight junctions. The connection between the anterior epithelial layer and the stroma via the dilator muscle fibres presumably restrains a separation of this epithelial layer from the iris stroma. However, spaces some $22-25 \mathrm{~nm}$ wide separate the two epithelial layers in places, and it must be presumed that this space is enlarged in the formation of a primary epithelial cyst of the iris, thus re-establishing an embryonic plane of separation.

Vail and $\mathrm{Merz}^{4}$ suggested that pigment cysts arise from a faulty developmental apposition of layers of
Fig. 6 Macrophotograph of collapse of the cyst into empty folds immediately after treatment.

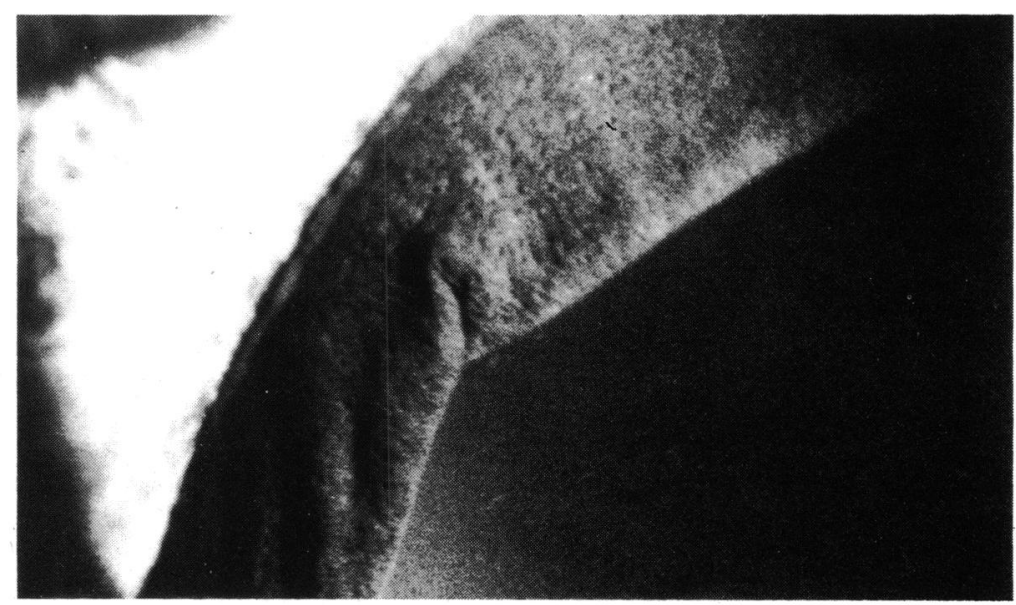


the iris epithelium (representing the inner and outer layers of the optic cup) at about the $110 \mathrm{~mm}$ or fourmonth stage of intrauterine development. ${ }^{5}$ The separation of these layers in utero, which was said to persist at the pupil margin up to the seventh month of intrauterine life (the marginal sinus of von Szily) is now regarded as an artefact. ${ }^{6}$ Nonetheless, the general hypothesis is attractive and is usually accepted. The suggestion that faulty apposition during the growth of the eye results from an abnormal traction of the zonule on the ciliary epithelium seems less secure except perhaps to explain peripheral iris cysts, which have an iridociliary location. Although most iris epithelial cysts are diagnosed in adults, this need not necessarily reflect growth. In a symptomatic patient diagnosis may be influenced by the nature of diagnostic techniques applied to young and old patients. In patients presenting with angle closure glaucoma it may be that continued growth of the lens in time pushes forward a pre-existing cyst until irisangle contact results. Shields, ${ }^{12}$ in his study of 59 patients with primary iris epithelial cysts, regarded them as stationary lesions which rarely progress. Nonetheless, he reported one instance of a midzonal pigment epithelial cyst which presented in a patient with iritis and disappeared in one week while the patient was being treated. This suggests that some typical 'primary' epithelial cysts may be acquired as a result of local ocular events. Secondary pigment epithelial cysts are described in aphakes, in association with congenital syphilis, and in response to topical therapy with ecothiopate iodide (Phospholine iodide) in children.

Shields ${ }^{1}$ has classified the primary cysts of the iris pigment epithelium as central (at the pupil margin), midzonal, and peripheral. Of these the peripheral are the most common (42 out of 59 patients in his series). They are distinguished by features in addition to those of position. The peripheral cysts are solitary, unilateral, more common in women (3:1), and present between the ages of 20-30 years (range 15-60 years) lying temporally more often than nasally in the iris. They usually transilluminate freely and may be almost transparent, which distinguishes them from the cyst studied here and makes it unlikely that our patient's cyst has arisen from such a source. Central and midzonal pigment epithelial cysts may be solitary or multiple, unilateral, or bilateral with similar clinical features apart from size and location. The larger midzonal cysts are most like the cyst described here except for size, and as in our case special techniques must be adopted to show transillumination. However, although the cystic space within a midzonal cyst may extend from the iris root to the area of the sphincter, in our patient it is evident that the cystic space extended to the pupil margin. Thus it may be necessary to regard the ring cyst as related to, though developmentally separate from, cysts described previously.

The differential diagnosis of a primary pigment epithelial cyst of the iris usually lies between an iris stromal cyst, secondary epithelial cyst, and an iris or ciliary body tumour. Both stromal cysts of the iris and iris tumours expand the iris stroma, whereas a pigment epithelial cyst if anything thins the stroma. A ciliary body melanoma does not transilluminate, and this can be demonstrated by observing the sclera in the region of the ciliary body while applying a transilluminator to the opposite limbus. Similarly, with a blue iris this technique may demonstrate lack of transillumination over the affected segment of iris. Iris transillumination does vary however. In the present case the diagnosis of a primary iris cyst was made on the basis of (a) smooth backward convexity of the pigment epithelial layer; $(b)$ apparent absence of the pigment epithelium in the iris plane on slit-lamp microscopy; (c) transillumination of all parts of the lesion; (d) normal transillumination features of the pars ciliaris/pars plana region; (e) a normal fluorescein angiogram without increased dye leakage; $(f)$ features of a cystic lesion with ultrasound.

In this study transillumination of the cyst was not observed by transpupillary slit-illumination nor by scleral illumination with a fibre-optic transilluminator. Punctate transillumination was demonstrated by directing the slit-beam through the iris stroma into the cyst while observing the pigment epithelium through the pupil. A similar technique using a contact lens has been recommended by Shields.?

The value of examining the iris pigment epithelial layer with the slit beam, where an epithelial cyst is suspected, does not seem to have been emphasised in the past. Where there was a light coloured iris the copper coloured line representing reflection from the posterior pigment epithelium was not visible, and instead a diffuse reflection of the same colour from the posterior wall of the cyst was dimly visible to one side of the reflection from the iris stroma itself. It is perhaps noteworthy that the posterior profile of the stroma in the slit beam overlying the cyst was still smooth, which suggests that the anterior epithelium was still present and made up the anterior wall of the cyst. This indeed is the histological appearance of other epithelial iris cysts.

Although the indications for ultrasonographic examinations of the anterior segment are not frequent, such procedures are helpful for assisting in the detection and differential diagnosis of cystic or tumorous lesions of the iris and ciliary body. Not surprisingly, the comparative rarity of these lesions is reflected by the small attention they have received in texts on ophthalmic applications of ultrasonography. ${ }^{?}$ 
For example, in a classification of common ophthalmic ultrasonic diagnoses based on 555 consecutive cases at the Case Western Reserve University School of Medicine, Purnell and Frank ${ }^{8}$ found the incidence of iris tumours to equal that of iris cysts at $0 \cdot 7 \%$. This small proportion of the total sample represented no more than $2 \cdot 1 \%$ of all anterior segment abnormalities examined by ultrasound in their sample. The preferred technique described by Coleman et al. ${ }^{9}$ for ultrasonic examination of the anterior segment involves using a water immersion bath to eliminate artifacts which occur near the transducer head. However, considerable improvements have recently occurred in transducer design such that the frequently distracting artifacts can be considerably minimised by using a liquid filled unit held in a liberal application of clear gel against the eyelids. Improved visualisation of the anterior segment structures have been described by Chang et al. "1" using high-resolution compound scanning with transducer frequencies of 15 to $20 \mathrm{MHz}$ and a narrowly focussed beam.

On examination by ultrasonography iris cysts and iris tumours may be differentiated by the presence or absence of echoes from the interior of these lesions. Cysts frequently arise from the posterior surface of the iris and appear as sonolucent or anechoic areas, whereas iris tumours appear as thickening of the iris. Ultrasonically, a tumour will appear solid or acoustically opaque, and when located in the iris it is usually possible to note extension into the angle, ciliary body, or the ora serrata.

Neither the mechanism for cyst formation nor the composition of the fluid content of the cyst are known. It was of interest that after laser photocoagulation of the pigment epithelial layer the ocular pressure rose. This might suggest that the fluid was of high viscosity, but it must also be noted that there were many cells in the anterior chamber, and the possibility exists that there was a transient cellular blockade of the angle. However, it is of note that the related pigment epithelial cysts of the ciliary body contain a mucopolysaccharide material, which is probably hyaluronic acid." The possibility exists that a fluid rich in hyaluronic acid was released into the anterior chamber at the time of treatment. The mechanisms of the angle closure glaucoma was assumed to be a peripheral closure of the drainage angle because of the forward bulk of the iris cyst and occlusion of the posterior chamber.

It was of interest that glaucoma attacks occurred while the patient was under treatment with low doses of pilocarpine, and some form of pupil block mechanism may be inferred. When the pupil was dilated for examination the ocular pressure did not rise, and the angle remained open. During mydriasis the epithelial cyst was distended centrally, and it may be that there was some mobility of the cyst during movements of the pupil such that it bulged forwards centrally when the pupil was dilated and bulged peripherally when the pupil was constricted. This 'rolling' action might make an additional contribution to the pupil block mechanism.

It was also of interest that the pupil at the time of presentation, when the ocular pressure was $58 \mathrm{mmHg}$, was dilated and round. Although this would appear to argue against a pupil block mechanism, and although at that stage a clear view of the iris was not obtained, it is a common experience in patients presenting with subacute rises in ocular pressure to high levels that a round pupil dilatation occurs. Thus it may be seen in subacute angle closure glaucoma (in patients presenting with haloes but little pain or redness of the eye) in rebleeds following a contusion hyphaema, where the pupil has originally been observed to be small and reactive, and where it is found to be dilated and round after clearing of a secondary total hyphaema, and finally, possibly in Urrets-Zavalia syndrome. It is suggested, therefore, that in this patient angle closure was precipitated when the pupil was in the relatively constricted condition, and that the pupil subsequently dilated as a result of the glaucoma.

Both xenon and laser photocoagulation have been used before in the successful treatment of pigment epithelial cysts of the iris ${ }^{4}$ and of secondary, epithelial, implantation cysts. ${ }^{12}$ No problem arose as a result of the entry of cyst fluid into the anterior chamber. The energy level used to penetrate the pigment epithelial layer was much lower than that required to produce a standard iridotomy via the iris stroma, but it is of a similar order to that used to rupture pigment epithelium left intact at a peripheral iridectomy site.

\section{References}

1 Shields JA. Primary cysts of the iris. Trans Am Ophthalmol Soc 1981; 79: 771-809.

2 Shields JA. Diagnosis and management of intraocular tumors. 1983. London: Mosby.

3 Hogan MJ, Alvarado JA, Weddell JE. Histology of the human cye. London: Saunders, 1971.

4 Vail D, Merz CH. Embryonic intraepithelial cysts of ciliary processes. Trans Am Ophthalmol Soc 1959; 49: 167-183.

5 Bernard J-A. Clay C. Photocoagulation an laser deskystes congenitaux du bood pupillaire. Bull Soc Ophtalmol Fr 1981; 81: 463-5.

6 Huidberg-Hansen J. Light neuroscopic studies of the marginal sinus (v. Szily) in the developing human eye. Albrecht von Graefes Arch Klin Ophthalmol 1971; 183: 134-43.

7 Coleman DJ, Lizzi FL, Jack RL. Ultrasonography of the eye. Philadelphia: Lea and Febiger, 1977

8 Purnell EW, Frank KE. Development and orientation of ophthalmic ultrasonography. In: Dallow RL ed. Ophthalmic ultrasonography. Comparative techniques. Boston: Little, Brown, 1979.

9 Coleman DJ, Dallow RL, Smith ME. Immersion ultrasonography: Simultancous A-scan and B-scan. In: Dallow RL ed. 
Ophthalmic ultrasonography. Comparative techniques Boston: Little, Brown, 1979.

10 Chang S, Dallow RL, Colcman DJ. Ultrasonic evaluation of intraocular tumours. In Jakobicc FA ed. Ocular and adnexal tumors. Birmingham, Alabama: Acsculapius, 1978.
11 Hogan MJ, Zimmerman LE. Ophthalmic pathology. London: Saunders, 1962.

12 Scholz RT, Kelly JS. Argon laser photocoagulation treatment of iris cysts following penetrating keratoplasty. Arch Ophthalmol 1982; 100: 926-7. 